Case Study

\title{
Effects of Individual Strengthening Exercises on Subdivisions of the Gluteus Medius in a Patient with Sacroiliac Joint Pain
}

\author{
WON-GYU Yoo ${ }^{1)}$ \\ 1) Department of Physical Therapy, College of Biomedical Science and Engineering, Inje University: \\ 607 Obangdong, Gimhae, Gyeongsangnam-do 621-749, Republic of Korea
}

\begin{abstract}
Purpose] We investigated the effects of individual strengthening exercises for subdivisions of the gluteus medius in a patient with sacroiliac joint pain. [Subject] A 32 year-old female who complained of pain in the posterior area of the left iliac crest and sacroiliac joints over a period of 6 months was the subject of this study. [Methods] She performed individual strengthening exercises for subdivisions of the gluteus medius over 3 weeks. Pain-provocation tests and VAS scores were evaluated before and after the intervention. [Results] After individual strengthening exercises for subdivisions of the gluteus medius, the subject showed no pain in the Gaenslen, Patrick, or REAB tests for the left sacroiliac joint. The VAS score was less the 3/10, compared with 7/10 initially. [Conclusion] Individual strengthening exercises for the subdivisions of the gluteus medius were effective at reducing SI joint pain for this patient.

Key words: Gluteus medius, Pain-provocation tests, Sacroiliac joint pain
\end{abstract}

(This article was submitted Feb. 3, 2014, and was accepted Mar. 31, 2014)

\section{INTRODUCTION}

Previous studies have established that the sacroiliac (SI) joint may be a source of low-back pain by demonstrating symptomatic relief after intra-articular injection into the sacroiliac joint ${ }^{1)}$. SI joint pain may occur when leaning forward to perform motions such as lifting, bending, or lowering that moves the center of gravity anterior to the acetabular axis ${ }^{2}$. SI joint pain has many causes, including inflammatory arthritis, ankylosis, osteoarthritis, and posttraumatic arthritis ${ }^{3}$. SI joint pain suggests that nociceptive and painful mechanical stress within the SI joint, or acting on the surrounding tissues attached to the innominate bones, is caused by pelvic asymmetry or SI joint instability $^{3)}$.

The gluteus medius controls femoral motion primarily during dynamic lower extremity motion and stabilizes the pelvis in the frontal and transverse planes ${ }^{4,5)}$. Weakness in or injury to the gluteus medius is associated with iliotibial band friction syndrome, SI joint pain, and low-back pain ${ }^{4)}$.

In clinical practice, therapists commonly suggest hip abduction exercises as a gluteus medius strengthening exercise for patients with SI joint pain. However, the gluteus medius muscle is segmented into three distinct portions: the anterior, middle, and posterior fibers of the gluteus me-

Corresponding author. Won-gyu Yoo (E-mail: won7y@inje. ac.kr)

(C) The Society of Physical Therapy Science. Published by IPEC Inc.

This is an open-access article distributed under the terms of the Creative Commons Attribution Non-Commercial No Derivatives (by-ncnd) License $<$ http://creativecommons.org/licenses/by-nc-nd/3.0/>. dius $^{6}$. Several studies have suggested that the three subdivisions of the gluteus medius can be activated in isolation ${ }^{6}$. Thus, in this study, we investigated the effects of individual strengthening exercises for the subdivisions of the gluteus medius in a patient with sacroiliac joint pain.

\section{SUBJECTS AND METHODS}

A 32 year-old female who complained of pain in the posterior areas of the left iliac crest and sacroiliac joints over a period of 6 months was the subject of this study. Ethical approval was obtained from the Yonsei University Faculty of Health Science Human Ethics Committee. The patient provided her written informed consent prior to participation in the study.

The subject experienced SI joint pain when bending at the waist. Additionally, severe pain occurred in the posterior areas of the left iliac crest and sacroiliac joints after standing for longer than an hour or after walking for more than $30 \mathrm{~min}$. Pain provocation tests reproduced pain in both sacroiliac joints through stressing. In pain provocation tests ${ }^{7)}$ for the left sacroiliac joint, the following tests yielded positive results: the Gaenslen test (the subject lies supine over the edge of a table and draws both legs up to the chest, then lowers the affected leg into hip full extension; pain indicates positive response); the Patrick test (the subject lies supine and places the affected-side foot on the opposite knee to achieve flexion, abduction, and external rotation of the hip while the examiner fixes the opposite anterior superior iliac spine (ASIS) and applies pressure to the subject's affected knee; pain indicates a positive response); and the resistive abduction (REAB) test (the subjects lies 
supine and performs $30^{\circ}$ abduction of the affected leg in hip full extension the pushes the affected leg to the side while the examiner holds the ankle; pain indicates a positive response) $)^{7}$. Our subject also experienced pain in the Gaenslen, Patrick, and REAB tests for the left SI joint. The patient described her pain on a visual analog scale (VAS), with ' 0 ' representing no pain and ' 10 ' the worst imaginable pain. A VAS score of $7 / 10$ was reported for the palpation state of the left iliac crest posterior areas in the prone position. Our patient performed individual strengthening exercises for the subdivisions of the gluteus medius over a period of 3 weeks. The anterior gluteus medius exercise was a left hip adduction exercise with knee extension in side lying. The middle gluteus medius exercise was a wall press exercise. The left middle gluteus medius was exercised by pushing the left knee, hip, and ankle at $90^{\circ}$ flexion against a wall and maintaining the contraction for $5 \mathrm{~s}^{6}$ ). The posterior gluteus medius exercise was the pelvic drop exercise, which is designed to maintain proper pelvic tilt. This exercise involves standing straight on an elevated platform and tilting the right pelvis while dropping the leg slightly lower than the left pelvis, and then pulling the pelvis slowly up using the gluteal muscles and hips without bending the knees $^{6)}$. For all exercises, three sets of 30 repetitions per day were performed for 3 weeks.

\section{RESULTS}

After performing the individual strengthening exercises for the subdivisions of the gluteus medius for three weeks, the subject showed no pain on the Gaenslen, Patrick, or REAB tests for the left sacroiliac joint, and her VAS score was less than $3 / 10$ during palpation of the left iliac crest posterior areas, compared with the $7 / 10$ initial score.

\section{DISCUSSION}

Sacroiliac joint dysfunction produces pain in a region similar to the referred pain pattern from the gluteus medius $^{8)}$. The gluteus medius is vital for lateral pelvis stabilization $^{8)}$. In fact, it is considered that the primary function of the gluteus medius is to stabilize the pelvis ${ }^{8)}$. Many therapists suggest that the best exercise for the gluteus medius is side-lying abduction. However, previous functional studies have found subdivisions of the gluteus medius, as suggested by previous anatomical studies ${ }^{6}$.
O'Sullivan et al. ${ }^{6}$ showed that activation of the gluteus medius was highest in the wall press exercise, and the posterior fiber showed the strongest activation during the pelvic drop, wall press, and wall squat exercises in healthy subjects. Thus, in this patient with sacroiliac joint pain, we assessed the effects of individual strengthening exercises for subdivisions of the gluteus medius. After performing the individual strengthening exercises for three weeks, our patient experienced no pain in the Gaenslen, Patrick, or REAB tests ${ }^{7}$, and her VAS score fell from $7 / 10$ to less than 3/10. Stability in the SI joint through the form and/or force closure mechanisms has been proposed as facilitating load transfer to the pelvis ${ }^{9)}$. A deficit in the form or force closure mechanism may be related to pain disorders of the lumbopelvic region ${ }^{9}$. In conclusion, individual strengthening exercises for the subdivisions of the gluteus medius were effective at reducing SI joint pain for this patient.

\section{ACKNOWLEDGEMENT}

This research was supported by Basic Science Research Program through the National Research Foundation of Korea (NRF) funded by the Ministry of Education, Science and Technology (No. 2012R1A1B4001058).

\section{REFERENCES}

1) Maigne JY, Aivaliklis A, Pfefer F: Results of sacroiliac joint double block and value of sacroiliac pain provocation tests in 54 patients with low back pain. Spine, 1996, 21: 1889-1892. [Medline] [CrossRef]

2) DonTigny RL: Pathology of the sacroiliac joint and its effect on norma gait. J Orthop Med, 2005, 27: 61-69.

3) Brolinson PG, Kozar AJ, Cibor G: Sacroiliac joint dysfunction in athletes. Curr Sports Med Rep, 2003, 2: 47-56. [Medline] [CrossRef]

4) Earl JE: Gluteus medius activity during three variations of isometric single-leg stance. J Sport Rehabil, 2005, 14: 1-11.

5) Schmits RJ, Riemann BL, Thompson T: Gluteus medius activity during isometric closed-chain hip rotation. J Sport Rehabil, 2002, 11: 179-188.

6) O'Sullivan K, Smith SM, Sainsbury D: Electromyographic analysis of the three subdivisions of gluteus medius during weight-bearing exercises. Sports Med Arthrosc Rehabil Ther Technol, 2010, 2: 17-25. [Medline] [CrossRef]

7) Zelle BA, Gruen GS, Brown S, et al.: Sacroiliac joint dysfunction: evaluation and management. Clin J Pain, 2005, 21: 446-455. [Medline] [CrossRef]

8) Neumann DA: Kinesiology of the musculoskeletal system: foundations for physical rehabilitation. St Louis: Mosby, 2009.

9) O'Sullivan PB, Beales DJ, Beetham JA, et al.: Altered motor control strategies in subjects with sacroiliac joint pain during the active straight-legraise test. Spine, 2002, 27: E1-E8. [Medline] [CrossRef] 